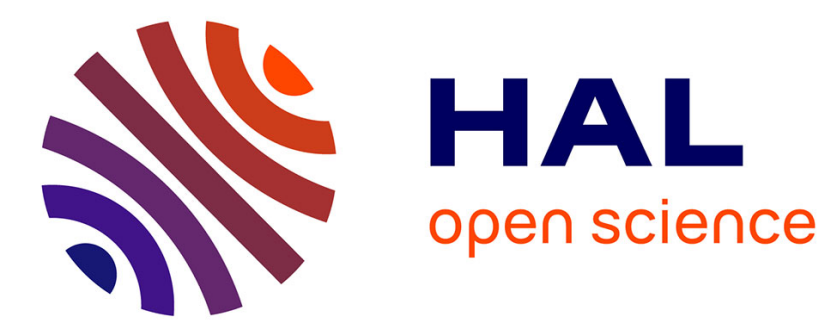

\title{
Faciès commerciaux et usages de la céramique à Lattes durant la Protohistoire
}

Eric Gailledrat

\section{To cite this version:}

Eric Gailledrat. Faciès commerciaux et usages de la céramique à Lattes durant la Protohistoire. Gallia - Archéologie de la France antique, 2008, Lattara/Lattes (Hérault) : nouveaux acquis, nouvelles questions sur une ville portuaire protohistorique et romaine, 65, pp.151-168. 10.3406/galia.2008.3338 . halshs-00371319

\section{HAL Id: halshs-00371319 \\ https://shs.hal.science/halshs-00371319}

Submitted on 19 Dec 2019

HAL is a multi-disciplinary open access archive for the deposit and dissemination of scientific research documents, whether they are published or not. The documents may come from teaching and research institutions in France or abroad, or from public or private research centers.
L'archive ouverte pluridisciplinaire HAL, est destinée au dépôt et à la diffusion de documents scientifiques de niveau recherche, publiés ou non, émanant des établissements d'enseignement et de recherche français ou étrangers, des laboratoires publics ou privés.

\section{(이) $\$$}

Distributed under a Creative Commons Attribution - NonCommercial - NoDerivatives| 4.0 


\title{
FACIÈS COMMERCIAUX ET USAGES DE LA CÉRAMIQUE À LATTES DURANT LA PROTOHISTOIRE
}

\author{
Éric GAILLEDRAT
}

\begin{abstract}
Mots-clés. Âge du Fer, céramique, amphores, vaisselle, commerce, faciès culturel.
Résumé. Au-delà des simples aspects typologiques, la céramique prise en tant qu'indicateur de la vie économique et sociale permet de mettre en lumière les phénomènes complexes qui rythment l'évolution de Lattes protohistorique. La période envisagée ici, entre la fin $d u \mathrm{VI}^{e}$ s. et la fin du II $s$. av. J.-C., montre avant tout l'importance fondamentale des liens, notamment commerciaux, entretenus avec Marseille grecque. Dans le même temps, si plusieurs signes témoignent de processus d'acculturation réels, la portée de ces derniers doit être nuancée en fonction du caractère fondamentalement indigène que revêt le site de Lattes jusqu'à la conquête romaine.
\end{abstract}

Key-words. Iron Age, pottery, amphorae, vessels, trade, cultural facies.

Abstract. Through pottery taken as an indicator of social and economic life, beside the typological features, we can gain insight into the complex phenomenon which punctuate the evolution of Protohistoric Lattes. The considered period, between the $6^{\text {th }}$ century to the end of the $2^{\text {nd }}$ century B.C., shows above all the fundamental importance of links, in particular trading contacts, with Greek Massilia. At the same time, even if several signs show real acculturation processes, their repercussions must be balanced according to the deep indigenous character of the site until the Roman conquest.

Translation: Isabelle FAUDUET

Schlüsselwörter. Eisenzeit, Keramik, Amphoren, Geschirr, Handel, kultureller Aspekt.

Zusammenfassung. Über den einfachen typologischen Aspekt hinaus gibt die Keramik als Indikator des wirtschaftlichen und sozialen Lebens Aufschluss über die komplexen Vorgänge, welche die Entwicklung des protohistorischen Lattes bestimmen. Die hier betrachtete Periode zwischen dem Ende des 6. und dem ausgehenden 2. Jh. v.u.Z. veranschaulicht vor allem die grundlegende Bedeutung der Beziehungen, insbesondere der Handelsbeziehungen, die Lattes mit dem griechischen Marseille unterhält. Wenn auch mehrere Anzeichen von reellen Akkulturationsprozessen zeugen, so muss deren Tragweite jedoch den grundsätzlich indigenen Zügen entsprechend nuanciert werden, die Lattes bis zur römischen Eroberung charakterisieren.

Übersetzung: Isa ODENHARDT-DONVEZ

Si la céramique constitue d'ordinaire, en contexte d'habitat, une donnée très largement utilisée pour appréhender les phénomènes économiques et sociaux, son importance à Lattes prend un relief particulier en raison de la masse documentaire acquise depuis le début des recherches menées sur le site, et plus particulièrement depuis le début de la fouille programmée entamée en 1983 dans le quartier de Saint-Sauveur, soit un corpus de plusieurs centaines de milliers de fragments. Il s'agit d'une base d'étude d'autant plus pertinente qu'elle est susceptible d'être traitée de manière statistique, une approche quantitative ayant été favorisée par la mise en place, dès le début des recherches programmées, de protocoles d'enregistrement rigoureux dans le cadre d'un système global de gestion des données de fouille («Syslat», cf. Lattara, 4 et 10). Le traitement statistique de ce mobilier repose donc sur la cohérence globale de l'enregistrement, et plus particulièrement sur l'application de méthodes de comptage uniformes se référant à un classement typologique des différentes catégories céramiques présentes en Méditerranée nordoccidentale, tel qu'il a été formalisé dans le dictionnaire DICOCER (Lattara, 6). 
La nature même du mobilier céramique présent à Lattes durant l'âge du Fer se révèle importante. Lieu d'échanges, ce type d'établissement portuaire voit en effet transiter des produits d'origines variées, parmi lesquels diverses importations méditerranéennes allant des amphores de transport à la vaisselle de luxe. Certaines d'entre elles, on pense notamment à la céramique attique, constituent des marqueurs chronologiques fiables et précis qui autorisent une datation fine des contextes stratigraphiques homogènes dans lesquels elles sont présentes. De la sorte, il est non seulement possible d'appréhender le faciès céramique lattois sur une longue durée, mais encore d'affiner l'analyse en fonction d'une échelle de temps de l'ordre du quart de siècle.

Lattara fait donc partie de cette chaîne d'établissements littoraux qui jalonnent le pourtour méditerranéen : lieux d'échanges au sens large du terme, lieux de rencontre entre individus d'origines diverses, où les phénomènes d'acculturation ont pu s'exercer de manière privilégiée. Dans le contexte géographique du golfe du Lion, fortement marqué par la colonisation phocéenne, le site de Lattes nous renvoie à une réalité multiforme qui est celle de l'emporion (Bresson, Rouillard dir., 1993). Mis en perspective avec les autres habitats protohistoriques du Midi méditerranéen, et notamment avec ceux de l'arrière-pays du Languedoc oriental, le gisement de Lattes constitue de fait une référence incontournable permettant d'appréhender tant l'évolution du faciès mobilier indigène que celle des courants commerciaux qui touchent cette partie du littoral méditerranéen, concernée à des degrés divers par le phénomène de la colonisation, notamment grecque. En effet, les problématiques soulevées ici par l'étude du faciès céramique se révèlent à la fois multiples et complexes.

Dans un contexte économique, et probablement politique, fortement marqué (au moins à partir du début du $\mathrm{V}^{\mathrm{e}} \mathrm{s}$.) par le poids de Marseille, la grande cité phocéenne pour ainsi dire voisine, l'évolution des pratiques de consommation perceptibles au travers de la céramique révèle un certain nombre de choix opérés par les habitants de Lattara. Ces choix s'inscrivent dans une logique d'acculturation, induite par l'existence de cadres sociaux particuliers. On se trouve en effet dans une situation de contact direct et continu entre des individus appartenant à des cultures différentes : indigènes d'une part, Étrusques, Grecs et autres peuples méditerranéens d'autre part.

Ces individus, ou ces groupes, sont présents à Lattes au sein d'une communauté dont le caractère indigène est patent dès les niveaux anciens du site, du moins à partir du deuxième quart $\mathrm{du} \mathrm{V}^{\mathrm{e}} \mathrm{s}$. Une situation plus contrastée existe en effet dans les décennies antérieures, où l'hypothèse d'une installation étrusque de quelque ampleur est actuellement envisagée. Les arguments permettant de déceler une situation de mixité, temporaire (présence occasionnelle de marchands étrangers) ou continue (installation durable de courtiers, éventuellement d'artisans, etc.) sont multiples tout au long de la période considérée, mais révèlent dans le même temps la complexité des phénomènes d'imitation et d'emprunt. Toutefois, de l'usage de l'écriture à celui de formes architecturales issues de registres méditerranéens, en passant par des habitudes de consommation allochtones, l'image obtenue n'est pas pour autant celle d'une société recevant de manière passive les influences externes.

L'ambivalence de ces phénomènes culturels et sociaux ressort logiquement de l'étude du faciès céramique lattois, dans la mesure où l'on touche ici au problème des modes de consommation. Le terme est bien sûr synonyme d' " usages », à savoir ceux de la vaisselle de table ou encore de la batterie de cuisine, mais il prend un sens plus prosaïque lorsqu'il s'agit de consommation des denrées acheminées dans les nombreuses amphores de transport mises au jour sur le site. Sur ce point, à la diversité (toute relative) des provenances répond l'omniprésence d'un produit importé particulier, en l'occurrence le vin, dont la consommation, répandue parmi les communautés occupant le Midi gaulois à l'âge du fer, trouve une faveur particulière sur certains sites littoraux parmi lesquels Lattara.

\section{LES PREMIERS TEMPS DE LATTES PROTOHISTORIQUE (VERS 510-450)}

Les données concernant les premiers temps de la vie de Lattes protohistorique demeurent certes lacunaires mais soulèvent, on l'a dit, la question d'une éventuelle fondation étrusque. Sans reprendre l'ensemble des arguments autorisant une telle proposition, et laissant de côté ses implications historiques, ces thèmes étant développés par ailleurs (voir Lebeaupin, Séjalon, supra, p. 45-64), on rappellera que le faciès céramique de cette période, couvrant l'intervalle 510-475 av. J.-C., apparaît particulièrement explicite. En effet, les ensembles homogènes disponibles à l'heure actuelle nous montrent un mobilier à très forte dominante étrusque (fig. 103) ; même si cette image résulte avant tout de l'abondance des amphores, la vaisselle étrusque est particulièrement bien représentée et côtoie d'assez nombreux vases non tournés (plus de $40 \%$ des fragments de vaisselle) qui, sur le plan typologique, s'inscrivent parfaitement dans 


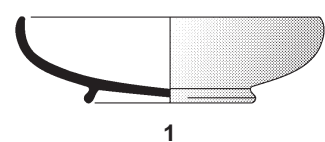

1

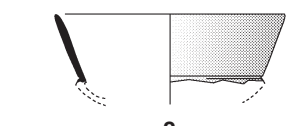

2

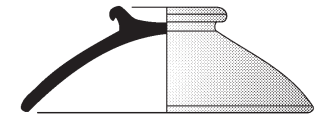

3

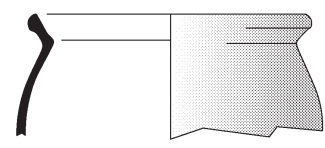

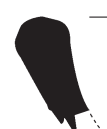
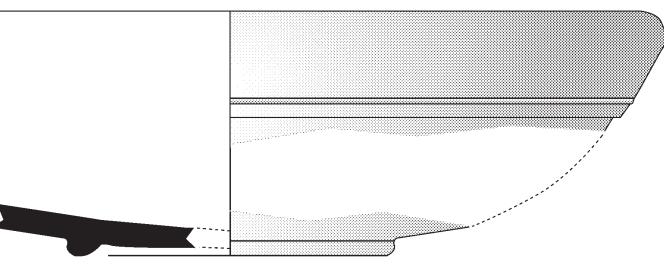

5
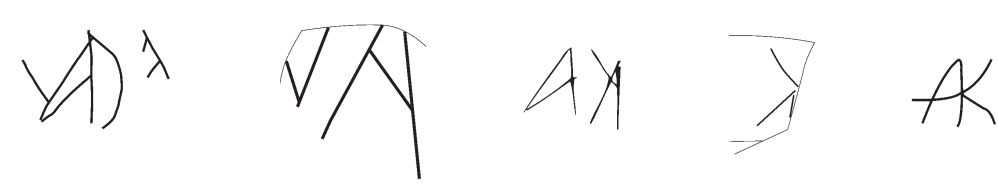

7

Fig. 103 - Mobilier céramique étrusque de Lattes (vers 510-450) : 1, 2, bucchero nero ; 3, 4, céramique de cuisine tournée ; 5, mortier; 6, amphore; 7, exemples de graffites en langue étrusque sur céramique commune (DAO : (C) UFRAL).

le répertoire indigène régional. L'ensemble est complété par quelques céramiques à pâte claire et attiques, dont la présence à cette époque renvoie de manière plus générale à la situation du Languedoc méditerranéen.

Cette prédominance de la céramique étrusque s'accompagne d'une forte représentation du matériel amphorique pris dans son ensemble, qui constitue près de $88 \%$ du total des tessons. Cette image, si elle doit être relativisée compte tenu de la faiblesse des surfaces fouillées, diffère en tout cas sensiblement de ce que l'on trouve par la suite (fig. 104). De tels taux laissent à penser que les quantités d'amphores vinaires constatées durant cette phase dépassent de loin les simples besoins locaux. Par ailleurs, ce mobilier est très largement dominé par l'amphore étrusque qui représente plus de $97 \%$ des tessons à l'intérieur de cette catégorie (fig. 105). Autrement dit, on est légitimement tenté d'interpréter ce faciès comme étant celui d'un site à forte vocation d'échange, avec possiblement un rôle de redistribution vers l'intérieur des terres. En effet, les pourcentages observés ne s'expliquent pas par la seule position littorale du site, mais invitent au contraire à reconnâ̂tre un faciès particulier. Force est néanmoins de constater que le contexte régional ne permet pas de surestimer cet éventuel rôle de Lattes en tant que point d'arrivée des produits étrusques en Languedoc oriental. Le panorama fourni à la même époque par les habitats indigènes de l'hinterland est en effet sensiblement différent (Py, 1990a, p. 518-607) : les apports étrusques, en diminution constante depuis le début $\mathrm{du} \mathrm{VI}{ }^{\mathrm{e}}$ s., sont alors non seulement faibles en quantité absolue, mais encore inférieurs aux importations massaliètes déjà bien représentées. À Lattes ces mêmes amphores de Marseille sont déjà présentes, mais elles se situent à un taux très nettement inférieur à celui de leurs homologues originaires d'Étrurie, essentiellement des amphores de type A-ETR 4 à pâte brune et dégraissant volcanique (fig. 103, $\mathrm{n}^{\mathrm{o}} 6$ ). Les autres productions amphoriques sont alors rarissimes (on pense notamment aux séries ibériques), voire absentes.

Cette singularité du faciès amphorique est accentuée par la répartition très particulière des différentes catégories de vaisselle. En effet, on trouve durant cette phase une forte représentation de produits étrusques aussi bien dans la vaisselle fine (bucchero nero tardif) que dans la vaisselle de cuisine, soit respectivement $7 \%$ et $34 \%$ des fragments. Outre cette proportion importante, la présence de bucchero nero est d'autant plus originale dans ce contexte que sont attestées des formes peu ou pas diffusées hors d'Étrurie, comme les bols de type B-NERO Bo4 (fig. 103, ${ }^{\circ} 1$ ). Les 


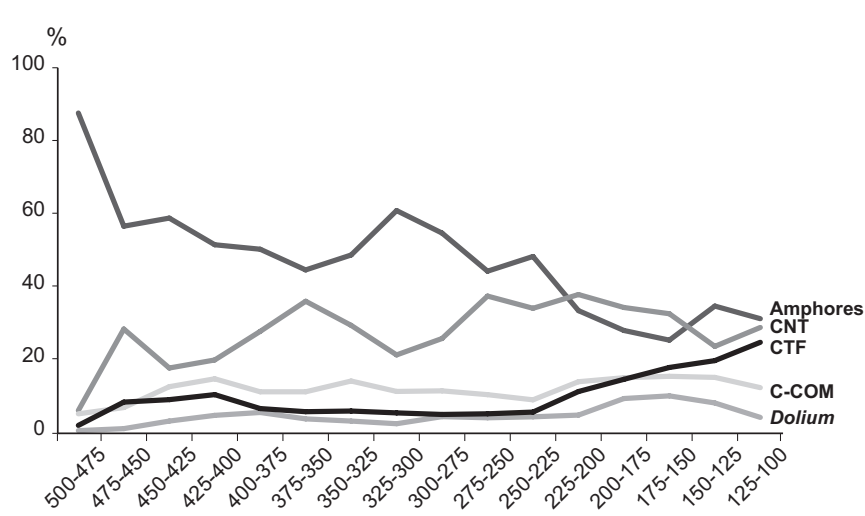

Fig. 104 - Répartition diachronique par grandes catégories du mobilier céramique de Lattes entre la fin du VIe s. et la fin du $I I^{e} s$. av. J.-C., exprimée en pourcentage sur le nombre total de fragments : CNT, céramique non tournée; CTF, céramique tournée fine; C-COM, céramique commune (DAO : E. Gailledrat, CNRS).

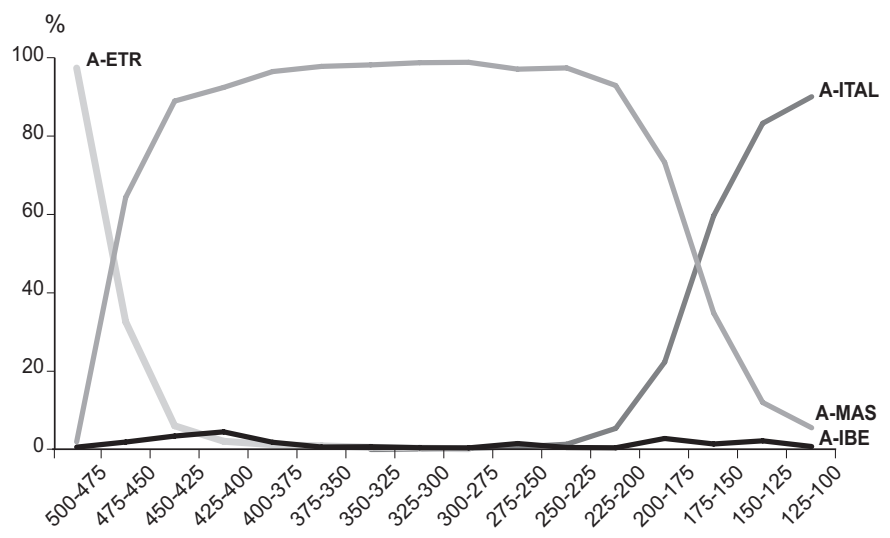

Fig. 105 - Répartition diachronique des principales catégories d'amphores présentes à Lattes entre la fin du VI ${ }^{e}$ s. et la fin du $I I^{e} s$. av. J.-C., exprimée en pourcentage sur le nombre de fragments d'amphores : A-ETR, amphore étrusque ; A-ITAL, amphore grécoitalique ou italique; A-MAS, amphore masaliète; A-IBE, amphore ibérique (DAO : E. Gailledrat, CNRS).

productions communes possèdent des pâtes semblables à celles des amphores présentes dans les mêmes contextes. Le répertoire des vases destinés à la préparation et à la cuisson des aliments accorde alors une place importante à l'urne ou « pot à cuire » ainsi qu'au mortier, tous deux étrusques (fig. 103, n 4 ). La préparation et la cuisson des aliments se font alors largement dans des formes importées. Le caractère " exotique » de ces dernières doit cependant être relativisé, la morphologie du pot à cuire étrusque étant, somme toute, très proche de celle de l'urne indigène utilisée aux mêmes fins.

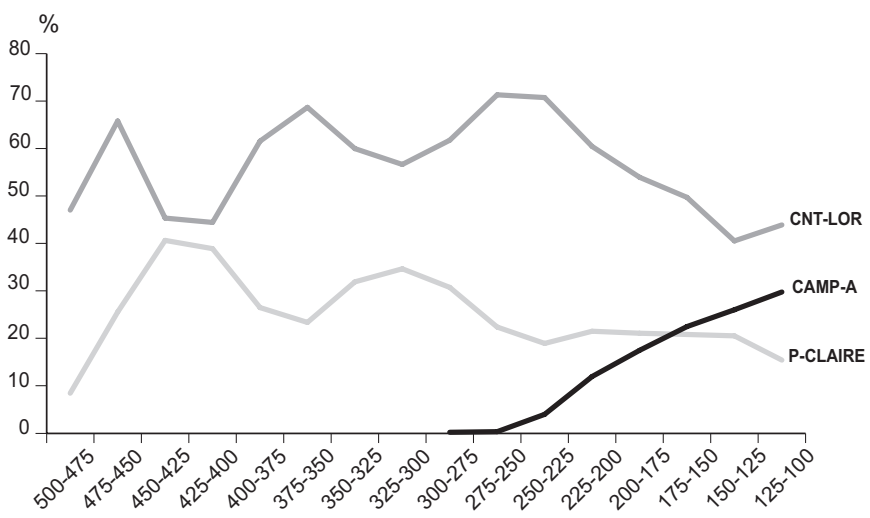

Fig. 106 - Répartition diachronique des principales catégories de vaisselle présentes à Lattes entre la fin $d u V^{e}{ }^{e}$ s. et la fin du $I^{e}$ s. av. J.-C., exprimée en pourcentage sur le nombre de fragments de vaisselle : CNT-LOR, céramique non tournée du Languedoc oriental ; P-CLAIRE, pâtes claires peintes ou non-peintes ; CAMP-A, campanienne A (DAO : E. Gailledrat, CNRS).

Dans la céramique non tournée indigène qui complète ce répertoire domestique, l'urne est prédominante et correspond à une forme utilisée tantôt pour le conditionnement, tantôt pour la cuisson des aliments. D'autres types sont également présents, notamment la jatte où la coupe, autrement dit les deux autres principales familles de vases constituant le répertoire régional.

Pour l'heure, le faciès céramique de Lattes à l'extrême fin $d u \mathrm{VI}^{\mathrm{e}} \mathrm{s}$. et au début $d u \mathrm{~V}^{\mathrm{e}}$ s. av. J.-C. apparaît donc comme une singularité, non seulement dans le contexte languedocien mais plus généralement dans celui du midi de la Gaule. Le décalage existant avec les habitats indigènes de l'intérieur des terres est patent et ne s'explique pas par la seule position littorale du site de Lattes. L'image que nous fournit la céramique de cette période plaide en faveur d'une présence étrusque, impression renforcée par l'existence de graffites en alphabet de même origine (fig. 103,

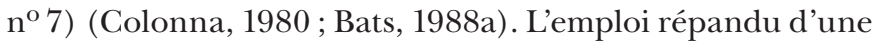
vaisselle de cuisine spécifique constitue un des meilleurs indices d'une telle présence, ou au moins d'un degré d'acculturation élevé, les habitudes culinaires étant considérées comme l'un des domaines culturels les plus réfractaires au changement (Bats, 1988c).

Il est dès lors important de remettre en perspective cette image avec celle d'un faciès mobilier où la composante indigène est bien présente, avec en l'occurrence une céramique non tournée de production locale ou régionale qui constitue une part significative de la vaisselle consommée sur le site (fig. 106). Certes, interpréter ces données en 
termes de cohabitation entre Étrusques et indigènes est sans doute prématuré. Une telle situation de proximité n'aurait pourtant rien de surprenant dans le cadre de ce que l'on perçoit être un établissement largement tourné vers les activités d'échange, et dont la fondation résulte sans doute d'une volonté commune émanant de ces deux composantes ethniques. L'image des premiers temps de la vie de Lattara reste à préciser, compte tenu de la faiblesse des surfaces fouillées, mais on mesure ici à quel point l'étude du faciès mobilier, en l'occurrence céramique, peut avoir un impact sur l'histoire de la colonisation dans cette partie de la Méditerranée.

\section{UNE PÉRIODE DE MUTATIONS PROFONDES (VERS 450-400)}

De nouvelles interrogations surgissent face aux changements significatifs qui interviennent dans le deuxième quart du $\mathrm{V}^{\mathrm{e}} \mathrm{s}$., période qui marque clairement une nouvelle étape dans la vie de Lattes protohistorique. L'apparente rupture déjà perceptible dans l'habitat proprement dit trouve un écho particulièrement explicite dans le faciès céramique.

Ces changements affectent en premier lieu l'orientation de l'activité marchande. Les taux d'amphores, s'ils diminuent durant cette période, n'en demeurent pas moins à un niveau élevé, avec environ $56 \%$ du total des fragments, chiffre qui se maintient à peu de choses près pendant tout le $\mathrm{V}^{\mathrm{e}} \mathrm{s}$. (fig. 104). Ce pourcentage doit encore être considéré comme important et caractéristique d'un site littoral, plus touché que les autres par les activités d'échanges liées aux trafics méditerranéens. Le décalage avec les habitats indigènes situés à l'intérieur des terres est en tout cas perceptible : les oppida de la région nîmoise, bien qu'assez largement concernés par les arrivages extérieurs, ne livrent alors qu'environ $25 \%$ de tessons d'amphores, soit à peine la moitié de ce que l'on trouve à Lattes durant la même période.

La nature des importations lattoises évolue également ; par rapport à la période précédente, se produit une inversion totale du ratio amphores étrusques/massaliètes (fig. 105). Ces dernières deviennent brusquement majoritaires, pour ne pas dire exclusives dans la mesure où elles constituent désormais près de $90 \%$ des fragments de cette catégorie, un taux qui se maintiendra à quelques nuances près jusqu'à la fin du $\operatorname{III}^{\mathrm{e}} \mathrm{s}$. av. J.-C. Cette omniprésence de l'amphore de Marseille à pâte micacée devient une des caractéristiques essentielles du faciès lattois, et traduit sans ambiguïté l'existence de relations commerciales privilégiées avec la cité phocéenne. Cette dernière semble désormais exercer une mainmise commerciale sur cette partie du Languedoc, comme le montrent non seulement les importations de Lattes mais encore celles des habitats de l'arrièrepays. L'opposition avec le faciès du Languedoc occidental (à l'ouest du fleuve Hérault) devient alors patente et révèle l'existence des deux aires commerciales et culturelles distinctes, l'une (Languedoc oriental) rattachée au domaine rhodanien, l'autre (Languedoc occidental et Roussillon) au domaine ibérique.

Ici encore se pose la question de savoir si Lattes a véritablement vocation à agir en tant que relais entre la Méditerranée et le monde indigène, ou si les taux élevés d'importation que l'on constate révèlent avant tout un faciès de consommation particulier, témoin d'un certain degré d'acculturation. Un regard porté sur les autres catégories céramiques, vaisselle de table et vaisselle de cuisine, nous apporte sur ce plan un certain nombre d'éléments de réponse.

En effet, l'évolution du faciès lattois au milieu du $\mathrm{V}^{\mathrm{e}} \mathrm{s}$. est sensible et dénote une acceptation plus grande qu'ailleurs des produits méditerranéens ou de leurs dérivés régionaux (fig. 107). L'exemple de la céramique à pâte claire illustre bien ce propos. Cette catégorie comprend en réalité un ensemble de productions, peintes ou non peintes, issues d'ateliers marseillais ou d'ateliers indigènes qui reprennent à leur compte, non seulement les techniques (celle du tour et celle de la cuisson en atmosphère oxydante), mais encore une partie du répertoire typologique et stylistique grec, dans ce cas associé à d'autres modèles plus spécifiquement indigènes. Le même processus de transfert et d'assimilation se retrouve d'ailleurs dans la céramique grise monochrome (fig. 107, n ${ }^{\text {os }} 10$ et 11), elle aussi dérivée de modèles originaires de Méditerranée orientale relayés en Gaule méridionale par la colonisation phocéenne. Cette dernière céramique ne connaît pas à Lattes le succès des productions à pâte claire, mais représente néanmoins une part non négligeable (entre $3 \%$ et $4 \%$ ) des fragments de vaisselle entre la seconde moitié du $\mathrm{V}^{\mathrm{e}} \mathrm{s}$. et le début $\mathrm{du} \mathrm{IV}^{\mathrm{e}} \mathrm{s}$.

À Lattes, dans la seconde moitié $d u \mathrm{~V}^{\mathrm{e}}$ s., les productions à pâte claire constituent environ $40 \%$ des fragments de vaisselle (fig. 106), autrement dit une part importante, pour ne pas dire essentielle, des vases présents sur la table. Durant cette période, les séries peintes à décor géométrique simple sont fréquentes et cohabitent avec des séries dépourvues de décor qui tendent à devenir de plus en plus nombreuses. L'essentiel des vases présents à Lattes semble, 

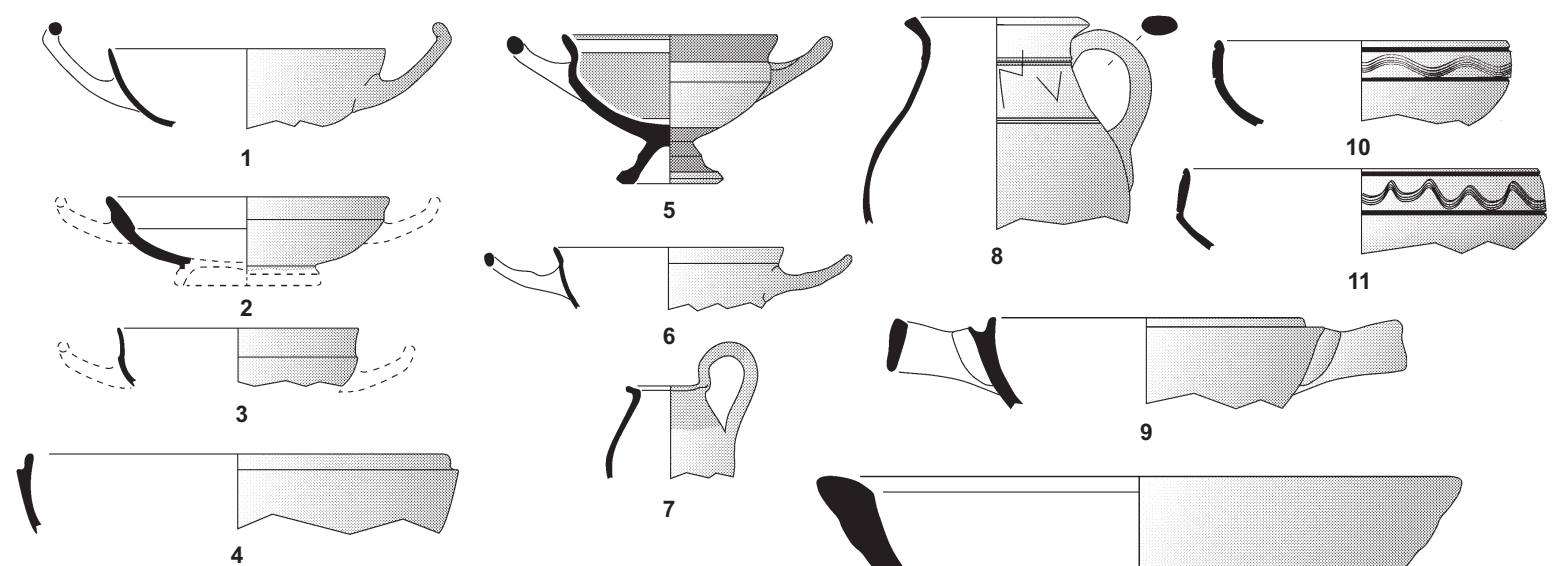

11
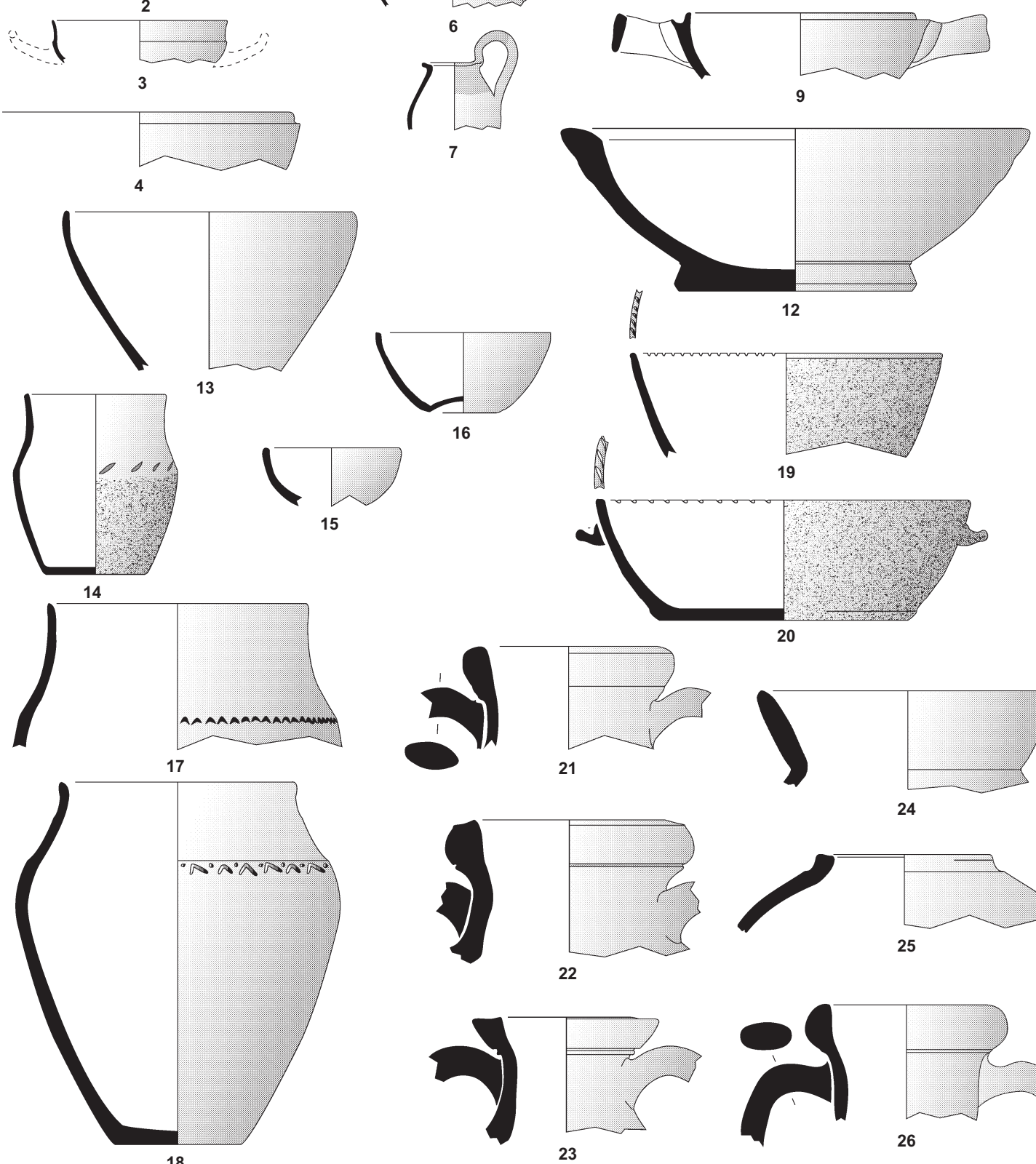

15

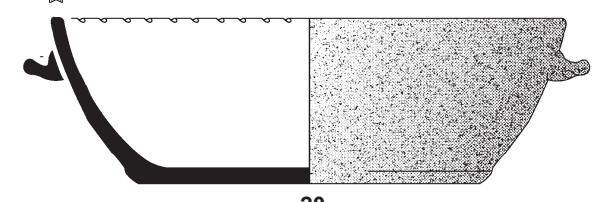

20

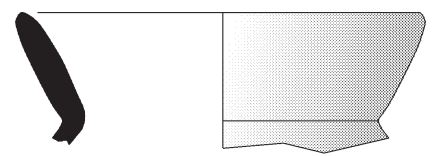

24
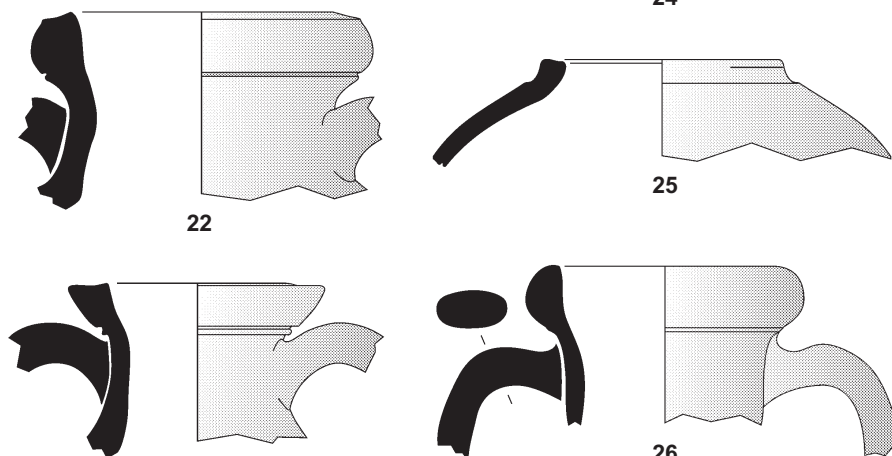

23
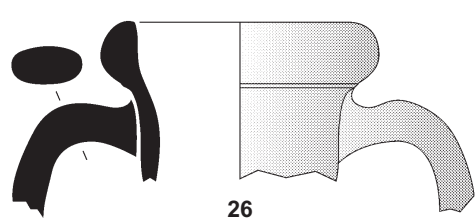

0

$10 \mathrm{~cm}$

Fig. 107 - Exemples de mobilier lattois de la seconde moitié du $V^{e}$ s. av. J.-C. : 1, attique à figures rouges ; 2-4, attique à vernis noir ; 5-9, céramique à pâtes claires peintes et non-peintes ; 10, 11, céramique grise monochrome : 12, mortier massaliète; 13-20, céramique non tournée du Languedoc oriental ; 21-23, amphore massaliète ; 24, amphore étrusque ; 25, amphore ibérique ; 26, amphore magno-grecque (DAO : ( $)$ UFRAL). 
en outre, de provenance proprement massaliète et paraît donc accompagner les autres productions originaires de la cité phocéenne telles que les amphores ou les mortiers de cuisine. L'hypothèse d'un artisanat céramique régional ne peut cependant pas être écartée, du moins pour une partie de ces séries dont le détail morphologique laisse entrevoir une certaine variété de centres de production. Il est clair cependant que cette céramique provient d'ateliers, sinon tenus par des Grecs, du moins fortement influencés par ces derniers, tant sur le plan technique que stylistique.

Le répertoire typologique des vases à pâte claire présents à Lattes (fig. 107, nos 5 à 9) n'offre pourtant pas toute la diversité de celui de Marseille, où cette céramique est non seulement d'un usage très courant dès le $\mathrm{VI}^{\mathrm{e}} \mathrm{s}$. av. J.-C. mais répond également à des modes de consommation spécifiques, propres à une cité grecque. Il n'en demeure pas moins que les formes attestées ici s'inscrivent fondamentalement dans un répertoire d'origine hellénique et non pas local, et que certains types rares en milieu indigène sont bel et bien présents, fût-ce de manière exceptionnelle. Ce répertoire comprend divers récipients à manger ou à boire et couvre une grande partie des fonctions liées à la table. Parmi ces dernières, le service et la consommation de boissons semblent occuper une place privilégiée. Les types génériques de la cruche et de la coupe à boire représentent ainsi l'essentiel des formes présentes dans la seconde moitié du $\mathrm{V}^{\mathrm{e}} \mathrm{s}$. Dans un cas comme dans l'autre - cruches à embouchure ronde et anse basse ou surélevée (CL-MAS 520-540) d'un côté, coupes à anses horizontales (CL-MAS 420) de l'autre -, il s'agit de formes spécifiquement grecques, reprenant des modèles ioniens ou attiques pour certaines d'entre elles. Ces vases destinés à verser des liquides et à les boire se retrouvent assez fréquemment en contexte indigène où ils accompagnent les amphores vinaires. Cependant, l'image fournie par la vaisselle lattoise est d'autant plus éloignée de celle d'un simple habitat indigène languedocien, qu'hormis les deux types génériques que l'on vient d'évoquer d'autres formes helléniques sont fréquentes et viennent compléter la gamme des fonctions occupées par cette vaisselle tournée. On pensera notamment aux coupes monoansées (CL-MAS 410), aux petites olpés piriformes (CL-MAS 420) et enfin aux mortiers (CL-MAS 620-630). Concernant ces derniers, la spécificité du faciès ne réside pas tant dans leur présence, mais bien plutôt dans leur fréquence, conjuguée au fait que des formes rares ou absentes des contextes indigènes contemporains sont ici attestées, ainsi la lékanis (CL-MAS 435), le lécythe (CL-MAS 550), le dinos (CL-MAS 581), etc.

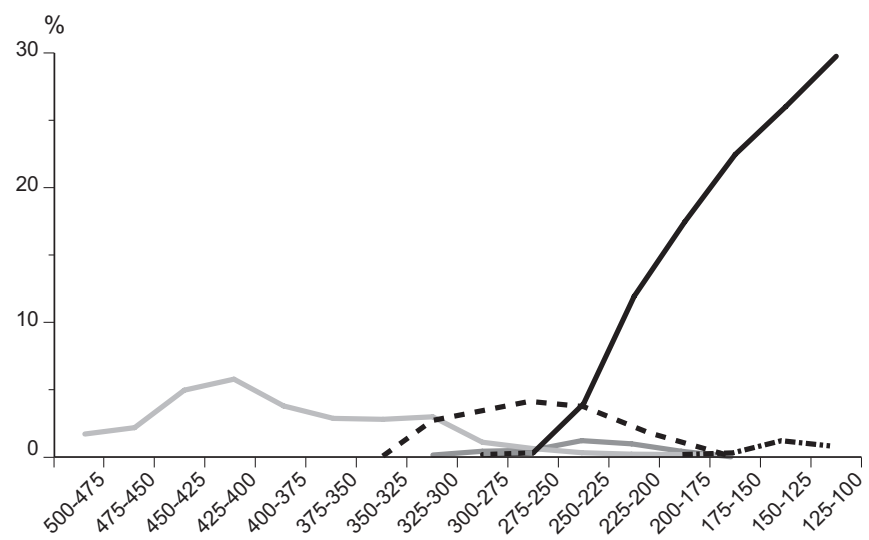

a

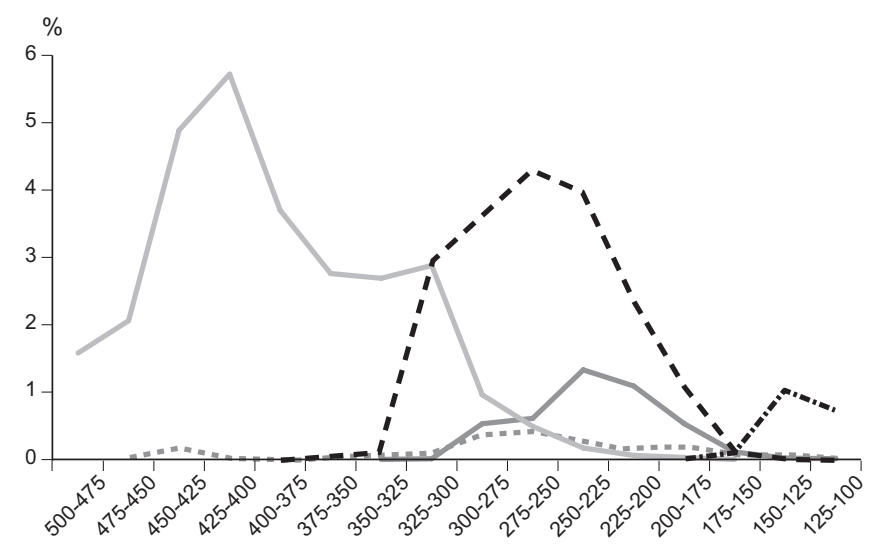

- - AUT-VN - CAMP-A - - CAMP-B - ROSAS - ATTIQUE - - PETEST

b

Fig. 108 - Répartition diachronique des principales catégories de céramique à vernis noir présentes à Lattes entre la fin $d u V^{e}{ } s$. et la fin du II $s$. av. J.-C., exprimée en pourcentage sur le nombre de fragments de vaisselle: $C A M P A$, campanienne $A ; C A M P-B$, campanienne $B$; AUT-VN, autres vernis noir ; ROSAS, céramique à vernis noir de Rosas ; PETEST, céramique de l'atelier des petites estampilles (DAO : E. Gailledrat, CNRS).

L'image fournie par la céramique attique accentue l'originalité du site de Lattes ; en effet, cette céramique fine représente en moyenne $5 \%$ des fragments de vaisselle dans la seconde moitié du $\mathrm{V}^{\mathrm{e}} \mathrm{s}$., avec un maximum dans le dernier quart de ce siècle (fig. 108). Lattes est donc non seulement plus touchée par ces arrivages que les sites de l'arrière-pays, mais on y constate une fois encore une variété plus grande des types présents. Même si les coupes sans tige (notamment à vernis noir) dominent très largement (fig. 107, n $^{\text {os }} 2$ et 3), répondant en cela à un faciès d'importation assez standardisé dont on trouve l'écho sur les autres sites de la région, 
d'autres formes plus originales en contexte indigène sont attestées en quantités notables, comme la lékanis (fig. 107, $\mathrm{n}^{\mathrm{o}} 4$ ). À la fin $\mathrm{du} \mathrm{V}^{\mathrm{e}}$ s., on constate une répartition sensiblement équivalente entre les vases à vernis noir et ceux à figures rouges. Parmi ces derniers, on retrouve divers types de coupe, avec ou sans tige (fig. 107, $\mathrm{n}^{\mathrm{o}} 1$ et fig. 109), et plus rarement le cratère.

Plus qu'une sélection au niveau de la demande, le faciès lattois révèle avant tout la réalité du panorama des exportations attiques vers l'Occident. La part qu'il faut attribuer aux choix de consommation à l'échelle locale reste globalement difficile à évaluer, en particulier lorsqu'il s'agit d'une catégorie de vaisselle importée telle que la céramique attique, redistribuée par des intermédiaires aussi divers que les Grecs de Marseille ou d'Ampurias, voire par d'autres négociants méditerranéens. En revanche, en prenant en compte l'ensemble des catégories de vaisselle présentes sur le site, on s'aperçoit que le vase attique trouve parfaitement sa place dans les pratiques locales en tant que complément des vases à pâte claire, parmi lesquels on a souligné la fréquence des coupes à boire et des cruches.

Cette image est assez éloignée de celle d'un strict faciès indigène et se rapproche au contraire de ce que l'on entrevoit pour Marseille même. La céramique utilisée à Lattes durant la seconde moitié du $\mathrm{V}^{\mathrm{e}} \mathrm{s}$. témoigne donc indubitablement d'un phénomène d'acculturation, qui ne peut s'expliquer ici que par l'existence de relations continues entre indigènes et négociants grecs, dans cet emporion situé dans l'orbite massaliète. Dans cet ordre d'idées, la consommation d'un produit spécifique comme le vin a pu favoriser l'usage de formes telles que la coupe à boire grecque qui accompagne l'amphore vinaire, et plus particulièrement celle de Marseille. Sans pour autant envisager de relations directes entre formes et fonctions (une coupe grecque ne sert pas nécessairement à boire du vin...), de tels usages témoignent en tout cas d'une «mode » dont l'intensité a indubitablement été plus forte ici qu'ailleurs.

L'intensité de cette acculturation doit bien évidemment être relativisée, ne serait-ce qu'en raison de la rareté des références autorisant des comparaisons avec le domaine phocéen d'Occident, Marseille en tête. Lattes reste avant tout un site indigène, dont le faciès céramique met en lumière une série d'emprunts au registre méditerranéen, mais témoigne dans le même temps de la permanence d'usages locaux. De ce point de vue, si la présence de vaisselle grecque ou de type grec révèle de manière générale un développement de l'usage des céramiques faites au tour, cela ne signifie pas pour autant une désaffection des productions modelées tradi-

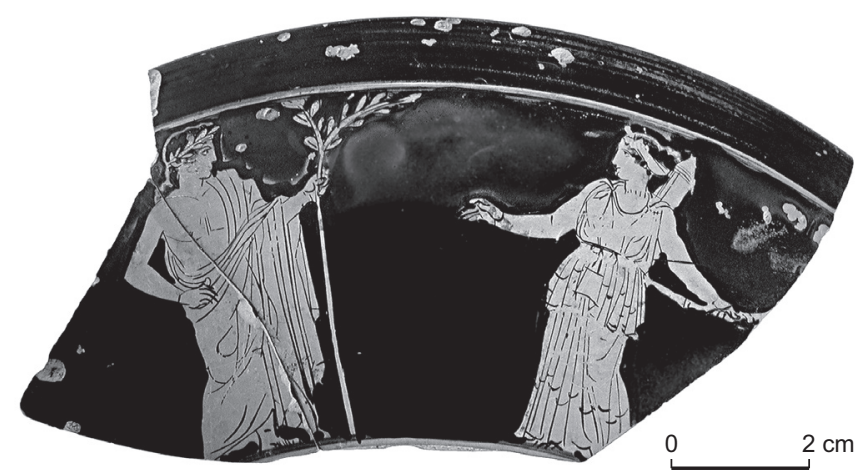

Fig. 109 - Coupe attique à figures rouges représentant Apollon et Artémis (vers 430-420) (photo : (C) UFRAL).

tionnelles, mais plutôt une évolution des fonctions liées à l'une et l'autre de ces séries. En effet, durant cette seconde moitié $\mathrm{du} \mathrm{V}^{\mathrm{e}} \mathrm{s}$., on constate également une augmentation globale des taux de céramique non tournée qui représentent désormais environ $40 \%$ des fragments de vaisselle. Cette évolution est avant tout la conséquence de la raréfaction des vases de cuisine étrusques, dont la présence est désormais résiduelle. De la sorte, même si les céramiques modelées se retrouvent encore à des degrés divers dans l'ensemble des fonctions (préparation, cuisson, service et consommation) et comme en témoigne l'existence d'un répertoire de formes relativement varié (fig. 107, nos 13 à 20), il semble en revanche évident qu'elles occupent de manière privilégiée le champ des céramiques de cuisine.

Par ailleurs, si les mortiers massaliètes font leur apparition au cours de cette période (fig. 107, $\mathrm{n}^{\mathrm{o}} 12$ ), les taux atteints sont loin d'égaler ceux atteints auparavant par les mortiers étrusques (fig. 103, no 5). De fait, rien ne permet de déceler pour cette période de quelconques pratiques étrangères aux habitudes culinaires strictement indigènes. L'emploi du mortier est de toute façon en soi un acte banal, et on imagine aisément que cette forme méditerranéenne, qu'elle soit grecque ou non, a logiquement trouvé sa place dans les foyers lattois ; l'objet en lui-même présentant des avantages sur le plan technique, probablement en raison de sa solidité.

L'apparition durant cette même période, et plus spécifiquement dans le dernier quart du $\mathrm{V}^{\mathrm{e}} \mathrm{s}$., d'urnes en céramique de cuisine tournée issues d'une production régionale (CCTLOR), ne modifie guère ce panorama. D'un point de vue fonctionnel, ces vases remplissent en effet exactement les mêmes fonctions que les urnes en céramique modelée. Ils témoignent malgré tout d'une certaine évolution du répertoire qui appelle la comparaison avec les usages alors en 
vogue dans le monde phocéen d'Occident, qu'il s'agisse de Marseille ou encore d'Ampurias où ce type de vase à cuire est effectivement bien représenté. Cela n'est d'ailleurs pas sans évoquer le répertoire étrusque dont Lattes nous donne un aperçu pour la fin du $\mathrm{VI}^{\mathrm{e}} \mathrm{s}$. et le début du V $\mathrm{V}^{\mathrm{e}} \mathrm{s}$, dans la mesure où, par excellence, la forme à cuire est ici encore une forme fermée, destinée à la cuisson par ébullition et mijotage, en l'occurrence une urne dont la morphologie a pu servir de modèle à ces mêmes vases tournés produits dans les ateliers indigènes du Languedoc.

Parallèlement, on note la rareté des pièces de cuisine en céramique commune grecque (non massaliète), terme qui recouvre en réalité un ensemble de productions relativement mal définies quant à leur origine exacte, mais qui sur le plan typologique affichent une identité méditerranéenne indéniable. Les formes en question, chytra et caccabé (pour bouillir et mijoter), lopas (pour mijoter et frire), tagénon (pour frire), complétées par des couvercles et des mortiers, renvoient effectivement à un répertoire hellénique. Leur présence en contexte indigène pourrait constituer un indicateur pertinent de l'adoption de pratiques de consommation exogènes (Bats, 1988c), voire de la présence physique de Grecs employant sur place les objets adaptés à leurs usages commensaux. Paradoxalement, cette batterie de cuisine semble peu utilisée sur les sites grecs d'extrême Occident, y compris à Marseille même, et on observe au contraire dans ces mêmes contextes un usage récurrent de productions régionales indigènes.

Ici comme ailleurs, impossible donc d'opposer de manière manichéenne une céramique de cuisine non tournée "indigène » à une céramique de cuisine tournée «grecque», et encore moins d'en faire un argument décisif permettant de reconnaître quelques Grecs installés à demeure dans le port de Lattes. Il faut peut-être considérer cette spécificité du répertoire phocéen d'Occident comme étant une conséquence quelque peu inattendue de l'acculturation résultant des contacts entretenus avec les sociétés indigènes. Quoi qu'il en soit, et au regard de la période précédente, on est tenté de reconnaître à Lattes une sorte de retour à des usages culinaires plus conformes à ce que l'on connaît en contexte languedocien. De la céramique de cuisine grecque présente à Lattes ressort l'image d'un site indigène concerné de manière réduite mais constante par l'arrivée, et l'usage, d'une vaisselle méditerranéenne. Dans ce cas précis, cette dernière est liée, du moins à l'origine, à des préparations culinaires assez spécifiques (ainsi celles faisant appel à l'huile d'olive), mais il est impossible de dire si celles-ci ont été reproduites par des Grecs résidant sur place, assimilées par certains individus ou groupes autochtones, ou si (plus simplement encore) les formes en question ont été détournées de leur destination première et incorporées à la «batterie de cuisine » locale, suivant probablement en cela l'exemple précédemment cité des mortiers.

Il va de soi que la nature des denrées consommées à Lattara alimente, sans jeux de mots, le discours qui peut être tenu sur cette lecture ethnographique du mobilier céramique. Sans rentrer dans le détail des résultats obtenus grâce à l'ensemble des études environnementales menées sur le site, l'image fournie par cette catégorie bien particulière de céramique que constituent les amphores de transport est elle-même porteuse de sens. On a vu à ce propos la place essentielle détenue par le commerce et la consommation du vin, étrusque tout d'abord, marseillais ensuite.

Le postulat de départ, communément admis, est que l'amphore massaliète est fondamentalement un conteneur vinaire (fig. 107, nos 21 à 23) : les autres contenus (olives, saumures de poisson, etc.) seraient plutôt liés à des réemplois, tandis que l'huile d'olive y semble absente (Bertucchi, 1992 ; Sourisseau, 1997). Les travaux menés dans les niveaux aquifères de Marseille ou dans des épaves ont par ailleurs montré la présence généralisée de poix sur la paroi intérieure de ces récipients. Il s'agirait d'une précaution destinée à préserver la qualité du contenu (dans ce cas, vin ou conserve de poisson, à l'exclusion de l'huile), mais on peut se demander dans quelle mesure la nature même du contenant, à savoir une amphore à pâte relativement poreuse de piètre qualité, n'a pas conditionné le choix de ce procédé technique. La question n'est pas anodine dans la mesure où la présence/absence de poix est souvent présentée comme étant un élément déterminant permettant l'identification d'une amphore vinaire (Sourisseau, 2004).

De fait, la question du contenu ne se pose pas véritablement pour les amphores de Marseille; on peut donc admettre que le vin est une boisson consommée régulièrement à Lattes, et ce dans des proportions non négligeables. En revanche, la place relative occupée par les autres catégories d'amphores pourrait le cas échéant indiquer des habitudes de consommation plus spécifiques. Ce serait par exemple le cas des amphores ibériques (fig. 107, no 25), quant à elles non poissées, et pour lesquelles se pose la question du ou des contenus multiples, y compris du vin, selon certains (Gailledrat, 2004), ou ayant principalement contenu de l'huile d'olive d'après d'autres, du moins en ce qui concerne les exemplaires mis au jour en Provence (Sourisseau, 2004). Dans ce cas, l'argument repose sur un constat simple, à savoir la présence régulière de ces amphores dans le courant 
$\mathrm{du} \mathrm{V}^{\mathrm{e}}$ s. en contexte grec, Marseille tout d'abord, mais aussi Arles, ce qui tendrait à montrer que les produits ainsi acheminés répondaient à des besoins spécifiquement helléniques, et qui en tout cas ne sont pas ceux des populations indigènes du Languedoc oriental ou de Provence où ces mêmes produits sont pour ainsi dire absents (Sourisseau, 2004). Sur ce plan, Lattes s'inscrit alors dans un schéma comparable à celui de Marseille, en ce sens que les amphores ibériques, bien que minoritaires, connaissent néanmoins un certain succès dans la seconde moitié du $\mathrm{V}^{\mathrm{e}} \mathrm{s}$. (fig. 105). Envisager que la consommation d'huile d'olive ait eu une certaine faveur à Lattes durant cette période irait dans le sens de l'hypothèse d'habitudes de consommation « à la grecque », que l'on pourrait associer aux quelques céramiques de cuisine évoquées précédemment et qui ne seraient pas incompatibles avec la présence de quelques Grecs résidant sur place. L'argumentation reste malgré tout d'autant plus délicate que ces amphores ibériques, rares en Languedoc oriental ou en Provence, sont en revanche abondantes en Languedoc occidental, sans que l'on puisse le moins du monde imaginer dans ces régions un goût immodéré pour l'huile, fût-elle ibérique. Résoudre cette contradiction en évoquant une probable différence de contenu entre les séries importées à Marseille, Arles, et probablement Lattes d'un côté, le domaine ibéro-languedocien de l'autre (Sourisseau, 1997, p. 205) n'est pas entièrement satisfaisant. On verra plus volontiers dans cette diffusion restreinte (géographiquement et chronologiquement) à l'est de l'Hérault la marque du dynamisme particulier des réseaux commerciaux phocéens en direction de la péninsule Ibérique. Leur présence non négligeable à Lattes durant cette période relève donc avant tout de la position littorale du site.

Dans la seconde moitié de ce siècle, et même si le poids de Marseille commence à se faire sentir de manière ostensible, Lattes est de toute évidence un port ouvert à des trafics multiples où se côtoient des individus et surtout des marchandises de diverses origines. Quelques amphores vinaires et oléicoles provenant de Grèce continentale ou de Grande Grèce sont donc logiquement présentes aux côtés des dernières amphores étrusques (fig. 107, nos 24 et 26). On trouve enfin de rares exemplaires d'amphores puniques, principalement issues des centres de production du détroit de Gibraltar. Plus généralement, il n'est pas anormal de trouver sur un site comme Lattes des produits à caractère « exotique », arrivant en quantités limitées, dans le cadre d'un commerce de redistribution sans commune mesure avec les arrivages à la fois massifs et directs en provenance de Marseille.

\section{LATTES ET LE MONOPOLE COMMERCIAL DE MARSEILLE (VERS 400-225)}

Durant cette période, l'image de Lattara en tant que port ouvert sur la Méditerranée se renforce apparemment. En témoignent notamment les taux d'amphores, à la fois élevés et relativement stables jusqu'au milieu du $\mathrm{III}^{\mathrm{e}} \mathrm{s}$., avec un maximum de $60 \%$ du total des fragments dans le dernier quart du IV ${ }^{\mathrm{e}}$ s. av. J.-C. (fig. 104). La diminution des importations constatée dans l'arrière-pays au cours de cette période (Py, 1990a, p. 559-561) ne concerne donc pas le site de Lattes, ce qui traduit bien l'existence d'un faciès de consommation particulier propre à cet établissement littoral où le vin reste un facteur important de la vie économique et sociale.

En outre, la tendance amorcée durant la séquence précédente, à savoir une orientation clairement massaliète du négoce, se voit ici renforcée. Entre 400 et 225 av. J.-C., les amphores de Marseille constituent ainsi en moyenne $98 \%$ des fragments de cette catégorie (fig. 105). Cette situation trouve d'ailleurs un écho dans l'arrière-pays, au point qu'est envisagée une situation de monopole commercial, exercé par les Marseillais sur un espace allant de la Provence au Languedoc oriental ; les régions situées à l'ouest de l'Hérault étant, quant à elles, dans une situation à la fois plus ouverte et plus intégrée à un circuit ibérique largement relayé par Ampurias. Conséquence logique de ces proportions écrasantes d'amphores massaliètes, les autres productions voient leur part relative diminuer d'autant ou stagner à des taux infinitésimaux. Les produits étrusques tendent à devenir résiduels, et ce courant s'éteint dans la première moitié du IV ${ }^{\mathrm{e}}$ s. Parallèlement, les apports ibériques qui ont connu une certaine faveur dans la seconde moitié du $\mathrm{V}^{\mathrm{e}}$ s. av. J.-C. décroissent brutalement durant l'intervalle 400-375 av. J.-C. Les autres amphores grecques ainsi que les produits d'origine punique sont alors pour ainsi dire anecdotiques.

Dans la mesure où les sites de l'arrière-pays ne reçoivent alors que de faibles quantités de produits marseillais, Lattes apparaît de fait, au même titre que les autres comptoirs lagunaires de la zone tels Le Cailar et Espeyran, comme un client privilégié de la cité phocéenne dans cette partie du Languedoc. En affinant l'échelle d'observation, les seules quantités d'amphores se révèlent malgré tout insuffisantes pour évaluer la part réelle de la consommation de vin à l'échelle locale (Py, 1999b). En revanche, la prise en compte du ratio entre surface fouillée/surface totale ainsi que la durée d'occupation du site met en lumière deux phéno- 


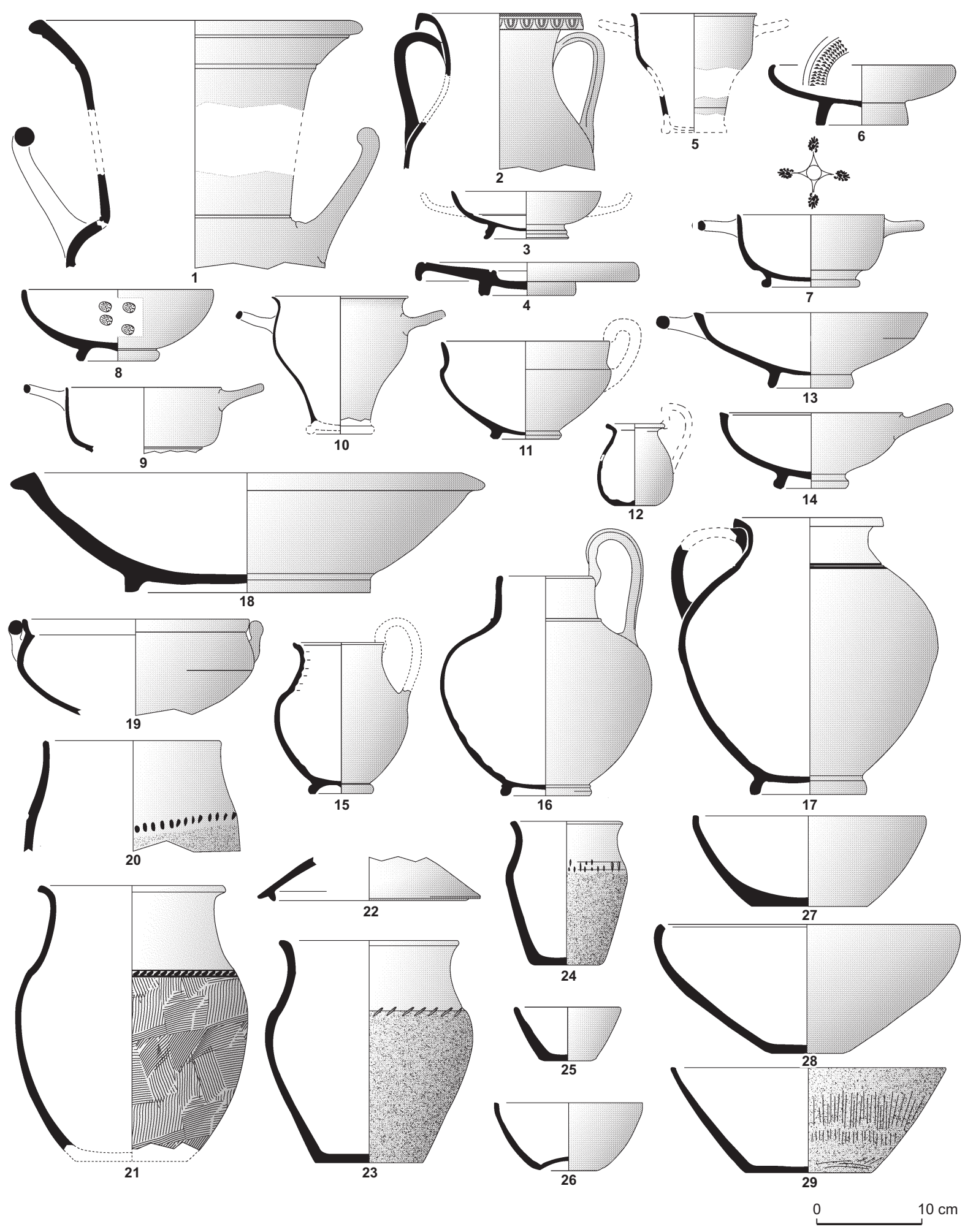

Fig. 110 - Exemples de mobilier lattois du IV s. av. J.-C. : 1-3, attique à figures rouges ; 4-7, attique à vernis noir; 8, atelier des petites estampilles ; 9, 10, pseudo-attique massaliète ; 11-17, céramique à pâtes claires ; 18, mortier massaliète ; 19, céramique commune grecque ; 20-29, céramique non tournée du Languedoc oriental (DAO : (C) UFRAL). 

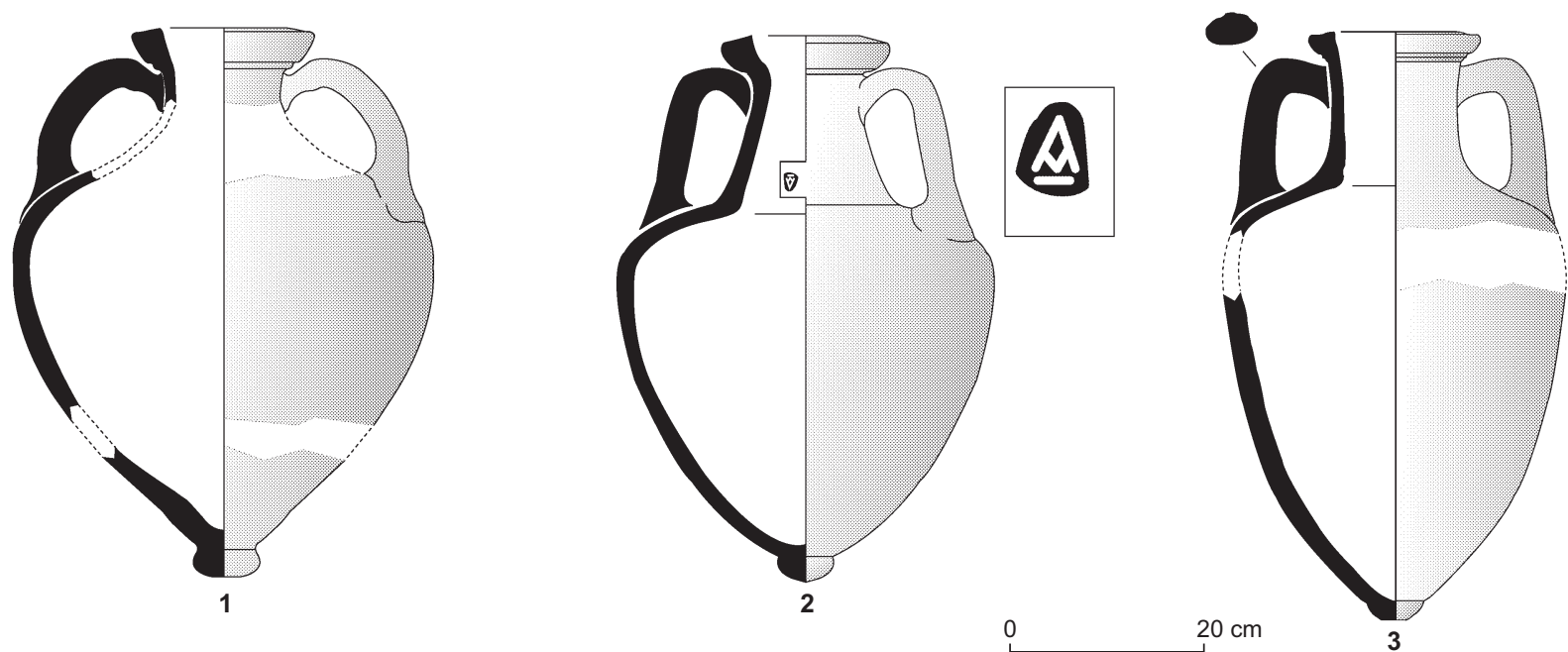

Fig. 111 - Exemples d'amphores massaliètes présentes à Lattes au IVes. av. J.-C. (DAO : (C UFRAL).

mènes indéniables. Le premier est l'aspect massif de cette consommation lattoise dans la mesure où, en extrapolant à l'ensemble du site les données disponibles, on obtiendrait pour le $\mathrm{IV}^{\mathrm{e}}$ s. un total de plus de 300000 amphores, représentant environ 7000 hectolitres de vin, soit presque $8 \%$ de la production de Marseille telle qu'elle a pu être estimée à partir du décompte des épaves de cette époque (Long, 1990). Le second phénomène est le décalage réel existant à ce niveau avec d'autres habitats de la région tels que le Marduel à Saint-Bonnet-du-Gard (Gard) ou Roque-de-Viou à Saint-Dionisy (Gard), où les quantités consommées sont estimées vingt fois inférieures (le Marduel) et cinquante fois inférieures (Roque-de-Viou) à celles de Lattes (Py, 1999b, p. 654-655). De telles estimations laissent bien sûr de côté la question, pourtant fondamentale, des modes de consommation. Or, le caractère communautaire de cette dernière au sein des sociétés indigènes a maintes fois été mis en avant (Dietler, 1999). Le vin, plus qu'une denrée courante absorbée quotidiennement, devient dans de tels contextes un élément à valeur sociale, lié à l'ensemble des pratiques et des événements rythmant la vie de la communauté. Il se développe alors à Lattes une habitude véritablement spécifique de consommation du vin, qui n'est déjà plus celle d'un simple milieu indigène.

Dans le même temps, le faciès céramique montre de manière plus globale cette importance du courant marseillais, qui ne se résume pas à l'importation d'amphores vinaires mais est également perceptible au niveau de la vaisselle, fine ou commune (fig. 110 et fig. 111). On constate, en effet durant cette période un usage toujours très répandu des pâtes claires, aux côtés desquelles on retrouve désormais un nouveau témoin de l'artisanat de la cité phocéenne, à savoir la céramique pseudo-attique (fig. 110, nos 9 et 10). Cette production à vernis noir fait son apparition dans le dernier quart $d u V^{e}$ s., mais c'est au début du siècle suivant qu'elle connaît une certaine faveur, du moins à Lattes, et ce jusque vers 300 av. J.-C. Malgré tout, et parallèlement à cette place privilégiée accordée à la céramique tournée, une évolution sensible du répertoire lattois est perceptible au début $\mathrm{du}_{\mathrm{IV}}^{\mathrm{e}} \mathrm{s}$. Cette évolution se traduit en particulier par une augmentation des céramiques modelées qui constituent alors plus de $60 \%$ des fragments de vaisselle, et jusqu'à $68 \%$ dans le deuxième quart du IV s. (fig. 106). Ce regain se fait notamment aux dépens de la céramique à pâte claire ; bien que toujours très présente, cette dernière constitue désormais moins de $30 \%$ du même total et à peine plus jusqu'au début du III $^{\mathrm{e}}$ s. Ce chiffre reste néanmoins important et montre l'ancrage profond de l'usage de ce type de vaisselle dans les habitudes lattoises.

Concernant ces productions, la tendance amorcée durant la période précédente, à savoir la raréfaction des séries à décor peint, s'accélère au début $\mathrm{du} \mathrm{IV}^{\mathrm{e}} \mathrm{s}$. L'évolution $\mathrm{du}$ répertoire est sensible (fig. 110, $\mathrm{n}^{\text {os }} 11$ à 17 et fig. 112 , $\mathrm{n}^{\text {os }} 7$ à 11), mais se place avant tout sur le plan typologique, les usages liés aux céramiques à pâte claire évoluant quant à eux relativement peu. Son utilisation en tant que vaisselle de table ressort de la diversité des pièces liées au service comme à la consommation individuelle d'aliments et de boissons. Le répertoire est assez étendu, même si 

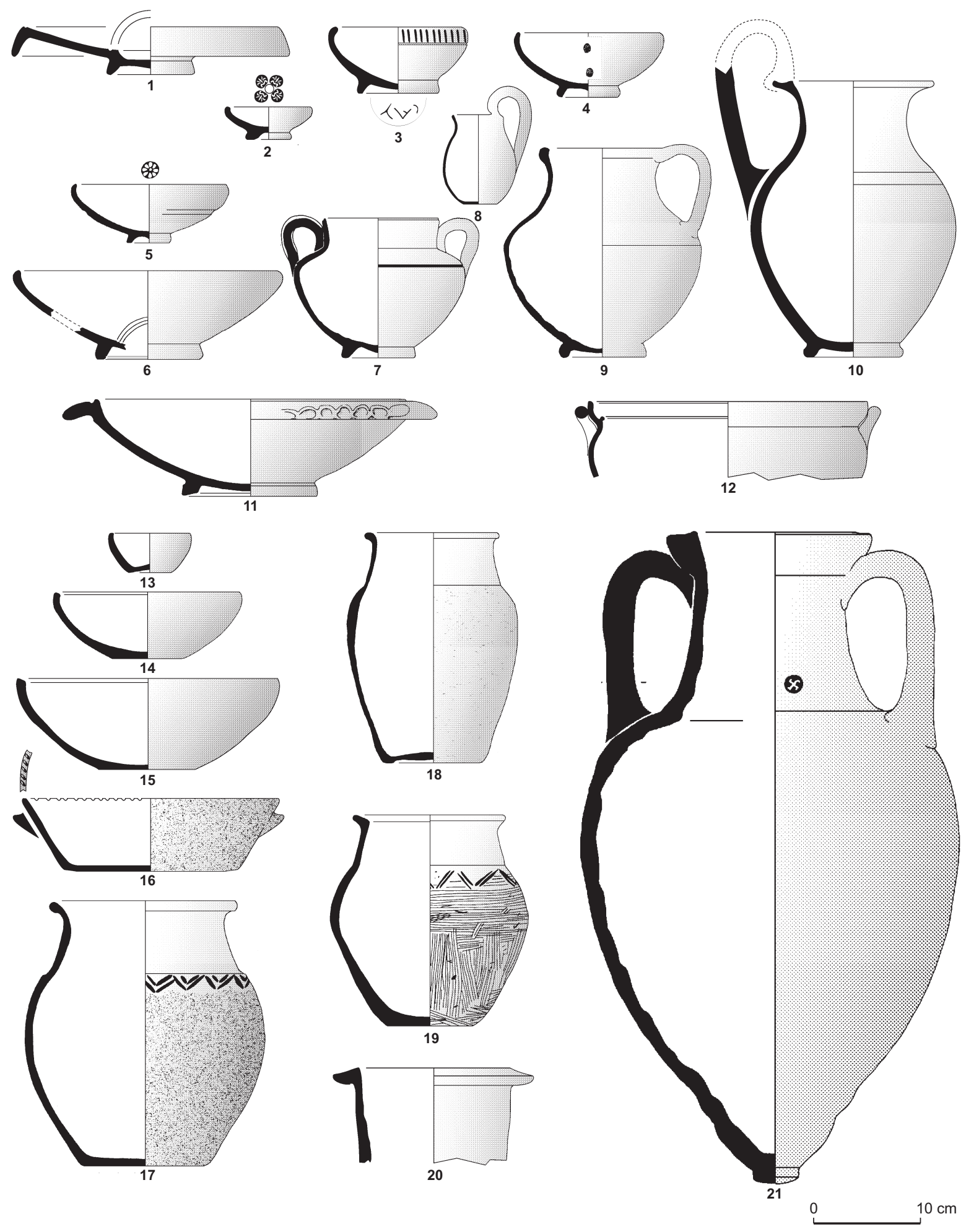

Fig. 112 - Exemples de mobilier lattois du III e s. av. J.-C. : 1, campanienne A ancienne; 2, 3, ateliers de Rosas ; 4, atelier des petites estampilles ; 5-10, pâtes claires ; 11, mortier massaliète ; 12, commune grecque ; 13-19, céramique non tournée du Languedoc oriental ; 20, amphore gréco-italique; 21, amphore massaliète (DAO : (C) UFRAL). 
certaines formes sont d'un usage courant et subissent peu d'évolutions morphologiques tout au long de la période concernée. Parmi les formes ouvertes, les coupes monoansées (CL-MAS 410) déjà présentes à la fin du V e s. sont désormais de loin les plus nombreuses (fig. 110, $\mathrm{n}^{\text {os }} 13$ et 14). Encore assez souvent munies d'un décor peint, ces coupes font vraisemblablement office de vases à manger plutôt qu'à boire. Elles accompagnent sur la table divers types moins bien représentés, ainsi les coupes sans anses à profil caréné (CL-MAS 222) ou diverses variantes de coupes et coupelles à profil continu (CL-MAS 230) dont la part tend d'ailleurs à augmenter au cours du $\mathrm{III}^{\mathrm{e}} \mathrm{s}$. Parmi les formes plus spécifiquement à boire, sont notamment présentes les coupes à anses dérivées de modèles attiques (CL-MAS 430) ou encore les gobelets à une anse (CL-MAS 450), quant à eux plus spécifiques du $\mathrm{IV}^{\mathrm{e}} \mathrm{s}$. D’autres emprunts au répertoire typologique attique sont notables, ainsi le plat à poisson (CL-MAS 121), qui prend place dans un service dont on mesure le côté déconnecté des standards purement indigènes. Les cruches, très souvent dépourvues de décor peint, sont une nouvelle fois nombreuses dans leurs différentes variantes typologiques (fig. 110, nos 15 à 17 et fig. 112, $\mathrm{n}^{\text {os }} 9$ et 10 ) et constituent alors près d'un vase en pâte claire sur deux. Enfin, les petites olpés (CL-MAS 521-522) sont non seulement encore présentes durant cette période mais tendent même à devenir de plus en plus nombreuses, tout en évoluant peu sur le plan morphologique (fig. 110, no 12 et fig. 112, no 8).

Les importations attiques, moins fréquentes que durant la seconde moitié du $\mathrm{V}^{\mathrm{e}}$ s., sont encore bien présentes durant les trois premiers quarts du $\mathrm{IV}^{\mathrm{e}} \mathrm{s}$. av. J.-C. La part relative des vases à figures rouges tend logiquement à décroître au profit des séries à vernis noir. De même que pour la céramique à pâte claire où l'on observe une répartition différenciée du répertoire entre séries peintes et non peintes, les formes représentées en céramique attique sont assez caractéristiques de l'une ou l'autre de ces séries, avec une évolution représentative de la mode des ateliers fonctionnant à cette époque. De la sorte, les vernis noirs sont avant tout représentés par des vases à boire. Pour le début $\mathrm{du} \mathrm{IV}^{\mathrm{e}} \mathrm{s}$. il s'agit principalement de coupes sans tige (stemless cups), qui cèdent la place dès le deuxième quart de ce même siècle au bolsal (AT-VN 532-561) (fig. 110, $\mathrm{n}^{\mathrm{o}} 7$ ) ainsi qu'au skyphos (AT-VN 350-354) (fig. 110, no 5) et au bol à lèvre déversée (AT-VN 777-808), cette dernière forme étant moins spécifiquement liée à la boisson. Parallèlement, la céramique à figures rouges livre un certain nombre de coupes, principalement sans tige (AT-FR Ky13 ou Ky14) (fig. 110, no 3), ainsi que des skyphoi (AT-FR Ky15 ou Ky16b). L'autre forme un tant soit peu courante est le cratère, principalement du type en cloche (AT-FR Cr4) (fig. 110, no 1). On note par ailleurs que les formes peu diffusées en milieu indigène sont ici plus rares qu'auparavant, de sorte qu'on ne trouve que quelques exemplaires de pélikés (fig. 110, no 2) et de lékanés à figures rouges. La consommation de vin, avec le cratère pour le mélanger et la coupe pour le boire, reste donc l'argument principal de ce courant d'importation attique.

La présence de séries à vernis noir massaliètes («pseudoattiques ») reflète cette utilisation préférentielle de la céramique fine grecque pour tout ce qui se rapporte à la boisson. Reprenant les modèles attiques non seulement sur le plan technique mais également sur le plan typologique (fig. 110, $\mathrm{n}^{\text {os }} 8$ à 10), le répertoire comprend en effet pour l'essentiel divers types de coupes à boire. Enfin, d'autres catégories de céramique à vernis noir viennent compléter cette partie du service de table. Largement moins représentées, elles concernent pour l'essentiel des vases appartenant à l'atelier latin des petites estampilles ou provenant d'ateliers encore méconnus de la péninsule Italique.

L'augmentation des taux de céramique non tournée a été évoquée. Ces productions indigènes témoignent de la permanence, voire de la résurgence, d'habitudes de consommation traditionnelles. Dans ce cadre, les formes présentes se résument toujours aux quatre grandes catégories typologiques que sont les urnes, les coupes ou écuelles, les jattes et les couvercles, représentées dans des proportions relativement stables tout au long de l'intervalle chronologique pris en compte (fig. 110, nos 20 à 29 et fig. 112, n ${ }^{\text {os }} 13$ à 19). On note que la proportion d'urnes (notamment de type CNT-LOC U5) est plus importante entre 400 et 325 av. J.-C., tandis que la répartition entre urnes et coupes s'équilibre au milieu du III $^{\mathrm{e}}$ s. Les coupes (fig. 110, $\mathrm{n}^{\text {os }} 25$ à 27), principalement à profil convexe et bord évasé (CNT-LOC C1) ou convergent (CNT-LOC C2), semblent faire office de vases polyvalents pour la présentation ou la consommation de nourriture, les jattes semblant, quant à elles, plutôt liées de par leur taille à la préparation ou au service des denrées (fig. 110, $\mathrm{n}^{\text {os }} 28$ et 29). Le fait notable est donc encore la place privilégiée accordée à la forme générique de l'urne, éventuellement complétée par un couvercle (fig. 110, no 22), en tant que vase à conditionner et à cuire.

La notion de «prix » plus ou moins élevé que l'on peut associer à telle ou telle catégorie céramique est bien évidemment délicate à manier, mais a dû en partie intervenir dans la composition du répertoire domestique lattois. S'il 
est probable, pour ne pas dire évident, qu'un vase attique figuré devait avoir plus de prix qu'une coupe non tournée, la gamme des possibles entre ces deux extrêmes doit être assez étendue. La notion de « luxe » doit ici être employée avec précaution, y compris pour la vaisselle fine importée, mais les proportions observées entre les différentes catégories invitent néanmoins à reconnaître une certaine échelle de valeurs. Pour le IV ${ }^{\mathrm{e}}$ s., les estimations proposées tendent à confirmer à la fois la facilité d'acquisition des vases à pâte claire et la relative rareté des vases attiques, notamment figurés, une famille lattoise ayant en moyenne dû acquérir moins de deux vases fins par an (Py, 1999b, p. 655). Réduire l'usage de la céramique non tournée aux seuls arguments économiques n'aurait pourtant guère de sens, et l'on imagine plus volontiers que, sur ce plan, des choix d'ordre pratique et d'ordre culturel sont intervenus de manière complémentaire.

L'exemple de la céramique de cuisine illustre bien ce propos, dans la mesure où l'on privilégie avant tout l'adéquation entre, d'une part la forme choisie (l'urne) et un mode de cuisson (par ébullition), d'autre part entre la fonction en elle-même (cuire) et les propriétés physiques du récipient (supportant les chocs thermiques). Le besoin d'autre chose que la céramique modelée n'existe pas en soi, et vu que la transcription de ces urnes en céramique tournée n'a pas été jugée utile (les proportions de céramique de cuisine tournée régionale régressent depuis le $\mathrm{V}^{\mathrm{e}} \mathrm{s}$.), on peut en déduire qu'un tel processus technique ne représentait en réalité aucun véritable progrès. Dans un tel schéma, l'utilisation d'une vaisselle de cuisine exogène (on pense évidemment à la céramique de cuisine grecque) ne peut dès lors qu'être qu'anecdotique, à moins de s'insérer dans une évolution globale des pratiques culinaires liée à des changements culturels plus profonds. Certes, la permanence de l'urne non tournée en tant que vase à cuire " type » présente une certaine ambiguité ; si à première vue elle répond à des modes de préparation typiquement indigènes, la convergence avec d'autres pratiques méditerranéennes ne laisse guère apparaître d'éventuelles évolutions internes. Quoi qu'il en soit, on ne constate pas à Lattes une quelconque généralisation de la vaisselle de cuisine tournée méditerranéenne, mais au contraire une continuité des usages ancestraux.

Dès lors, l'image que nous donne la céramique de Lattes durant cette période permet de s'interroger sur l'évolution plus globale de cette société indigène, depuis longtemps déjà au contact du monde classique méditerranéen. En effet, si l'on met en perspective la situation telle qu'elle se présente dans la seconde moitié $d u \mathrm{~V}^{\mathrm{e}} \mathrm{s}$. avec celle que l'on vient d'évoquer pour le $\mathrm{IV}^{\mathrm{e}} \mathrm{s}$. et une partie du $\mathrm{III}^{\mathrm{e}} \mathrm{s}$., deux phénomènes successifs semblent se dessiner.

Le premier est, dans le courant $d u \mathrm{~V}^{\mathrm{e}}$ s., la relative perméabilité de la société lattoise aux influences extérieures. Celles-ci se manifestent par l'intrusion, sinon brusque du moins rapide, d'éléments de culture matérielle méditerranéenne. C'est le cas de la vaisselle tournée (notamment les céramiques à pâte claire), de la consommation de vin, et plus généralement de la relative diversité des produits et de la faveur particulière alors accordée à la céramique attique. Durant cette étape que l'on peut qualifier de « dynamique », la composante indigène, tout en maintenant certains usages identitaires, emprunte notamment au monde grec un certain nombre de pratiques de consommation. Celles-ci ne sont qu'en partie réinterprétées au travers d'un filtre qui est celui de la culture indigène. Ainsi, la vaisselle tournée utilisée à Lattes est pour l'essentiel une vaisselle importée : à l'inverse de ce que l'on observe en Languedoc occidental, on ne perçoit pas d'artisanat régional particulièrement actif qui développerait un répertoire hybride, associant les techniques grecques à un répertoire formel mêlant modèles allogènes et indigènes. Si production régionale tournée il y a, celle-ci se cantonne pour ainsi dire au répertoire marseillais. De même, et cette remarque vaut pour l'ensemble de la séquence chronologique prise en compte, les céramiques non tournées « indigènes » ne révèlent quant à elles que de rares emprunts typologiques aux répertoires méditerranéens.

Évoquer une société indigène passive face à ces influences externes serait exagéré. Il n'en demeure pas moins que l'évolution du faciès lattois durant l'intervalle 400-325/300 av. J.-C. est révélatrice de l'instauration de nouveaux rapports avec l'extérieur (ici la Méditerranée) et, plus précisément, avec Marseille. L'activité économique de Lattes est à l'évidence florissante, ce qui conforte bien évidemment l'intérêt des négociants grecs pour cette position commerciale. Cette dernière constitue en outre un relais en direction de l'ouest, à un moment (fin du $\mathrm{V}^{\mathrm{e}}$ s.-début $\mathrm{du} \mathrm{IV}^{\mathrm{e}} \mathrm{s}$.) où plus loin dans cette direction est fondée Agathé (Agde) dont Strabon laisse entendre qu'elle marque une certaine limite du « domaine » massaliète (Strabon, Géographie, IV, 1, 5).

De manière quelque peu paradoxale, au $\mathrm{IV}^{\mathrm{e}} \mathrm{s}$., l'ouverture de la société lattoise en direction du monde grec semble se figer sur certains points. Symptomatique est le fait que la vaisselle grecque ou de type grec ne s'immisce pas plus avant dans les pratiques de consommation indigènes. 
À l'inverse même, on constate un léger regain dans l'usage des céramiques non tournées traditionnelles. De même, la quasi-exclusivité du vin marseillais pose la question du choix opéré (ou non) à ce niveau par les habitants de Lattara. Sans dire pour autant que leur horizon méditerranéen se résumait aux navires arrivant de Marseille, on n'en demeure pas moins assez éloigné de l'image d'un port aux accents bigarrés, pour se rapprocher à l'inverse d'un habitat évoluant de manière quelque peu déconnectée de son contexte indigène languedocien.

\section{LE COURANT ITALIQUE (VERS 225-100)}

Si le début du $\mathrm{I}^{\mathrm{er}} \mathrm{s}$. av. J.-C. constitue une limite quelque peu artificielle pour évoquer le cas de Lattes protohistorique, la fin du $\operatorname{III}^{\mathrm{e}} \mathrm{s}$. constitue en revanche une nouvelle période charnière dans l'évolution socio-économique du site, ceci dans un contexte régional lui-même en évolution.

Cette période voit en effet l'arrivée, puis le développement très rapide des importations italiques, et plus particulièrement des amphores («gréco-italiques », puis « italiques » à partir du dernier quart du $\mathrm{II}^{\mathrm{e}} \mathrm{s}$.), ainsi que des céramiques à vernis noir et plus spécialement de la campanienne A.

Ce courant est, on l'a évoqué, amorcé au IV ${ }^{\mathrm{e}} \mathrm{s}$. avec les céramiques à vernis noir de l'atelier des petites estampilles. Il connaît une nouvelle étape après le milieu du III $^{\mathrm{e}} \mathrm{s}$., époque à laquelle on repère les premières campaniennes $\mathrm{A}$ anciennes. Celles-ci deviennent rapidement plus nombreuses et constituent déjà $12 \%$ du total des fragments de vaisselle dans le dernier quart du $\mathrm{III}^{\mathrm{e}}$ s., pour croître ensuite de manière régulière jusqu'à la fin $d u \mathrm{II}^{\mathrm{e}} \mathrm{s}$. où elles représentent jusqu'à $30 \%$ du total (fig. 106).

Les arrivages amphoriques suivent cette tendance. Les amphores gréco-italiques apparaissent en effet au même moment que les campaniennes A anciennes et progressent de manière relativement similaire. On perçoit néanmoins un certain décalage chronologique, dans la mesure où la période de forte croissance des arrivages se situe non pas durant l'intervalle des années 225-200 mais bien plutôt dans le premier quart du II ${ }^{\mathrm{e}}$ s. av. J.-C. (fig. 105). Ces importations de vin italien se font aux dépens des productions de Marseille. Les amphores gréco-italiques deviennent ainsi majoritaires à Lattes entre 175 et 150 av. J.-C., avec près de $60 \%$ des fragments d'amphores, et occupent à la fin $\mathrm{du} \mathrm{II}^{\mathrm{e}} \mathrm{s}$. (avec $90 \%$ du même total) une place relative comparable à celle qu'occupaient les produits marseillais un siècle plus tôt. Si les amphores massaliètes se raréfient assez rapidement, on constate malgré tout que le vin italien est d'abord consommé sur le littoral ou sur les sites grecs (y compris à Marseille même) avant d'être diffusé vers l'intérieur. En définitive, cela ne fait que refléter un schéma de consommation " méditerranéen » en vigueur à Lattes depuis longtemps. La diffusion des céramiques communes de type italique reste pourtant minoritaire. Une fois encore, on ne perçoit pas à ce niveau un quelconque accroissement de l'imprégnation du fond culturel local.

$\mathrm{Si}$ le répertoire de la campanienne A ancienne est encore peu étendu, on assiste durant le $\mathrm{II}^{\mathrm{e}} \mathrm{s}$. à une diversification importante de celui-ci. Les formes ouvertes, bols et assiettes, prédominent très largement (fig. 113, $n^{\text {os }} 1$ à 7). Il est alors symptomatique qu'une telle répartition se fasse parallèlement à la raréfaction des coupes en céramique non tournée. À l'inverse des séries à vernis noir des phases antérieures, la campanienne A devient alors d'un usage véritablement courant et concurrence fortement sur les tables lattoises la céramique à pâte claire, qui connaît de nouvelles évolutions typologiques. La céramique non tournée reste elle aussi d'un usage commun, même si la tendance est à la régression de cette production face aux séries tournées. Il s'agit toujours de la principale catégorie de vaisselle utilisée à des fins culinaires.

En marge de ce répertoire quelque peu stéréotypé, dominé très largement par les catégories qui viennent d'être évoquées, on constate durant cette phase, et plus particulièrement dans la seconde moitié du $\mathrm{II}^{\mathrm{e}} \mathrm{s}$. av. J.-C., une certaine diversification de la vaisselle présente sur le site. On pense notamment à la céramique de type celtique (fig. 113, $\mathrm{n}^{\circ} 8$ ) ou encore aux communes ibériques (fig. 113, $\mathrm{n}^{\text {os }} 10$ et 11 ), et surtout aux productions grises de la côte catalane (fig. 113, no 12 ) qui représentent de $4 \%$ à $5 \%$ de l'ensemble des fragments de vaisselle durant cette période. Parallèlement, le regain constaté au niveau des amphores ibériques (qui, pour l'essentiel, proviennent également de Catalogne) entre 200 et 125 av. J.-C., permet de reconnaître l'existence d'un courant méridional non négligeable. Les deux phénomènes observés à Lattes, d'un côté le développement des importations d'amphores gréco-italiques, de l'autre celui (certes plus réduit) de produits originaires du nord-est de la péninsule Ibérique, sont sans doute en partie liés, du fait de l'ouverture précoce d'Ampurias à ces mêmes produits italiques. Les Marseillais eux-mêmes semblent bien avoir joué un rôle dans cette diffusion (fret de retour maritime ?) qui, à l'image de ce que l'on observe durant les périodes antérieures, s'appuie sur une diffusion essentiellement littorale. 

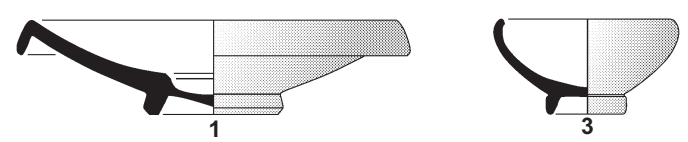

*2
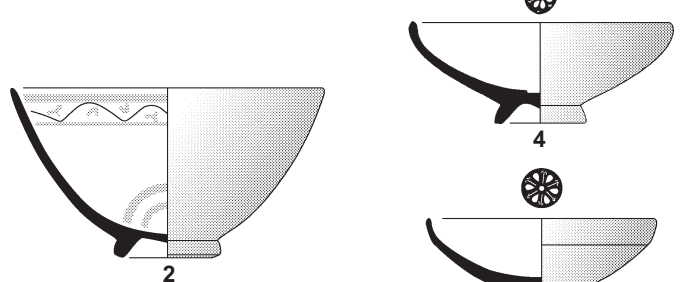

8
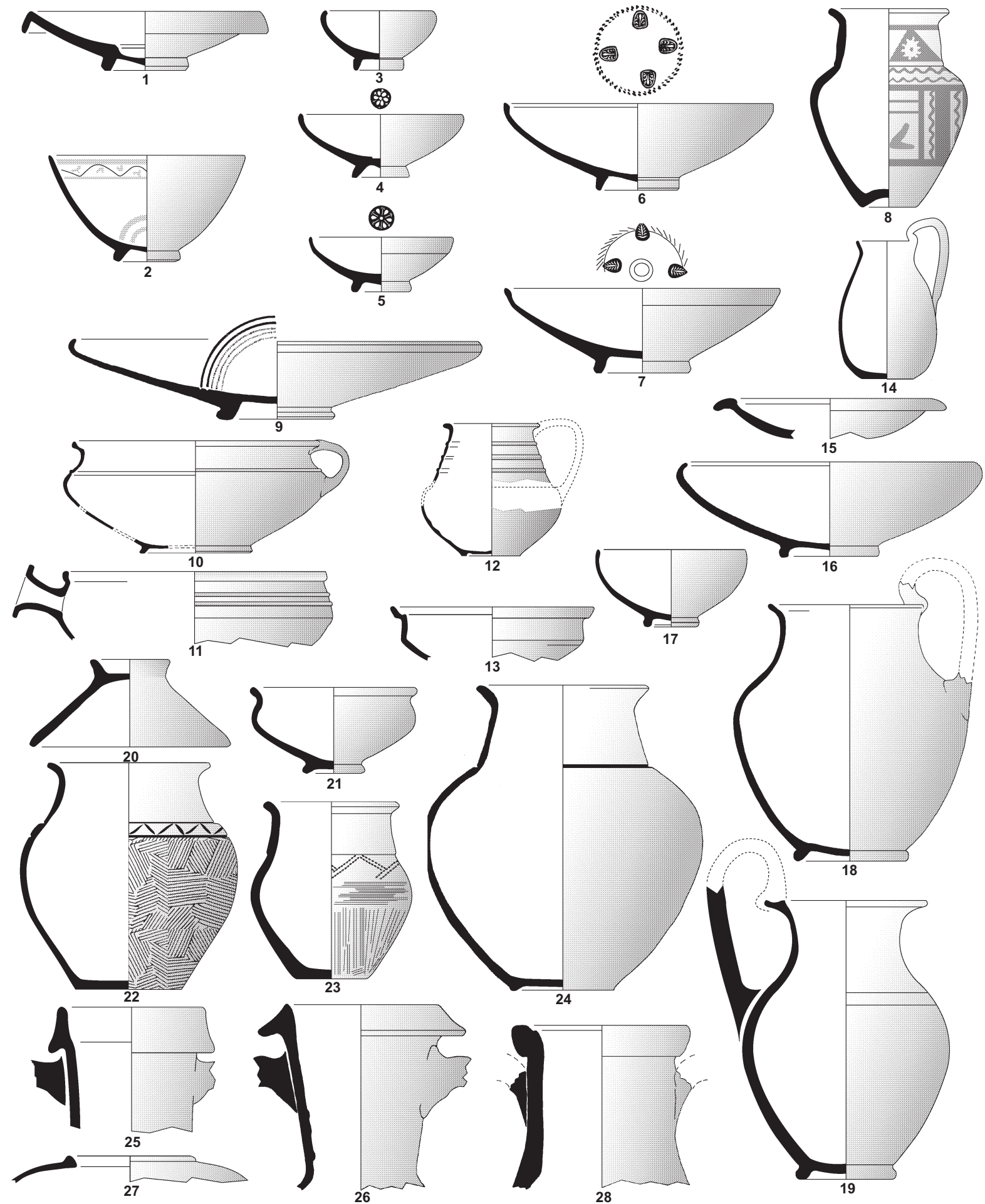

28

$10 \mathrm{~cm}$

Fig. 113 - Exemples de mobilier céramique lattois du II ${ }^{e}$ s. av. J.-C. : 1-7, campanienne A ; 8, celtique peinte ; 9, campanienne B ; 10, 11, commune ibérique; 12, grise de la côte catalane; 13, commune italique; 14-19, pâte claire ; 20-24, céramique non tournée du Languedoc oriental ; 25, 26, amphore gréco-italique ; 27, amphore ibérique ; 28, amphore massaliète (DAO : (C) UFRAL). 
Le II ${ }^{\mathrm{e}}$ s. av. J.-C. connaît d'autres évolutions perceptibles au travers du mobilier céramique. On relève ainsi la multiplication des dolia. Ces grands récipients sont présents depuis le milieu du $\mathrm{V}^{\mathrm{e}} \mathrm{s}$. à des taux à la fois bas et relativement stables, entre $2 \%$ et $5 \%$ du total des fragments, avec toutefois un premier pic à la fin $d u V^{\mathrm{e}} \mathrm{s}$. Au début du $\mathrm{II}^{\mathrm{e}}$ s. av. J.-C. en revanche, on constate une brusque augmentation de ces taux qui atteignent alors près de $10 \%$ du même total. De tels vases servent d'ordinaire à contenir des céréales, voire de l'huile en contexte méditerranéen. Mais à Lattes, leur multiplication peut aussi s'expliquer par le développement d'une viticulture locale. Divers indices en témoignent : en premier lieu, les prélèvements carpologiques effectués dans les niveaux de cette époque montrent que les pépins de raisin appartenant à des espèces cultivées se multiplient de manière sensible (Py, 1990a). L'utilisation de ces jarres en tant que conteneurs à vin ou cuves de fermentation n'aurait alors rien de surprenant mais, pour l'heure, aucune structure de production n'a été identifiée sur le site même. L'hypothèse semble en tout cas validée par la récente mise en évidence dans les environs immédiats de l'agglomération protohistorique (fouille préventive de "Port Ariane » à Lattes) de trous de plantation de vigne datés de la fin du $\mathrm{III}^{\mathrm{e}}$ s.-début du $\mathrm{II}^{\mathrm{e}} \mathrm{s}$. av. J.-C. (Daveau dir., 2007).

À ce moment, les habitants de Lattara expriment encore cette dualité perceptible depuis plusieurs siècles, entre ouverture et résistance aux apports externes. Des techniques méditerranéennes de mise en culture et de transformation des produits (ici la vigne et le vin) sont acquises de manière précoce. Dans le même temps, Lattes n'a jamais autant utilisé de vaisselle importée, et pourtant, une fois encore, les gestes associés à la céramique (avec notamment ce désintérêt pour les céramiques de cuisine tournées italo-grecques) témoignent de la permanence d'habitudes indigènes. 